\title{
Topical or oral fluralaner efficacy against flea (Ctenocephalides felis) transmission of Dipylidium caninum infection to dogs
}

\author{
Deepa Gopinath', Leon Meyer ${ }^{2}$, Jehane Smith² and Rob Armstrong ${ }^{3^{*}}$ (D)
}

\begin{abstract}
Background: Dipylidium caninum is a common tapeworm of dogs contracted from ingestion of fleas containing the infective cysticercoid stage. Fluralaner is a systemically distributed isoxazoline class insecticide that delivers highly effective activity against fleas and ticks for up to 12 weeks after a single oral or topical treatment. This study evaluated the impact of this flea insecticidal efficacy on the transmission of $D$. caninum to dogs.

Methods: Dogs were weighed and treated with a cestocide and then randomly assigned to 3 groups of 8 . Fluralaner was administered topically (at the commercial dose) to one group and orally to another group while the third received topically administered sterile water. All dogs were subsequently infested with about $100 \mathrm{D}$. caninum infected Ctenocephalides felis at 7, 14, 21, 28, 35, 42, 49, 56, 63, 70, 77 and 83 days after treatment. Visual proglottid inspections and counts were conducted daily from 35 to 113 days post-treatment. Post-treatment $D$. caninum incidence was calculated for each group and compared between treated and untreated groups.
\end{abstract}

Results: All 8 dogs in the placebo-treated group became infected with $D$. caninum while no shed proglottids were observed at any point during the post-treatment period from any dog in either fluralaner treated group.

Conclusions: The insecticidal efficacy of a single treatment of either orally or topically administered fluralaner prevented D. caninum transmission from infected fleas to susceptible dogs for up to 12 weeks following administration.

Keywords: Dipylidium caninum, Dogs, Fluralaner, Flea control, Flea-borne disease

\section{Background}

Dipylidium caninum, commonly known as the flea tapeworm, is a frequently diagnosed intestinal cestode parasite of dogs and cats, although occasionally humans have become infected following ingestion of the saliva of infected pets [1]. Ctenocephalides spp. fleas, of which C. felis is the most prevalent on domestic dogs and cats [2, 3], are intermediate hosts in the life-cycle of $D$. caninum. In brief, $D$. caninum eggs passed in the feces of infected animals are ingested by flea larvae in the environment and the flea larvae then develop into pupae while hosting tapeworm embryos. The adult flea then emerges and infests a host, and within 2-3 days the hexacanth cestode embryo develops into an infective cysticercoid stage within the flea. The cysticercoid larvae require at least 24-36 hours before

\footnotetext{
* Correspondence: robert.armstrong@merck.com

${ }^{3}$ Merck Animal Health, 2 Giralda Farms, Madison, NJ 07940, USA

Full list of author information is available at the end of the article
}

they are infective for the definitive host [4-6], a development period that is temperature-dependent [5]. Dogs and cats become infected when they ingest fleas containing the infective cysticercoid larvae during grooming. Adult $D$. caninum develop in the small intestine and within 2-3 weeks begin to shed egg packets called proglottids [7]. The overall pre-patent period can be as short as two weeks [6].

Clinical signs in D. caninum infected dogs generally consist of mild gastrointestinal manifestations and anal pruritus, which may cause the animal to exhibit 'scooting' behavior [8]. In addition, slowly motile proglottids may be observed on the animals' feces and the combination of this behavior and the sight of proglottids can be distressing for owners [9]. The potential zoonotic transmission of this parasite $[6,10,11]$ and wide geographical range [12] underline the value of protecting dogs and cats from $D$. caninum. Routine cestocidal treatment of household $\operatorname{dog}(\mathrm{s})$ and cat(s) is one option for managing this tapeworm [9]; however, the

(c) The Author(s). 2018 Open Access This article is distributed under the terms of the Creative Commons Attribution 4.0 International License (http://creativecommons.org/licenses/by/4.0/), which permits unrestricted use, distribution, and reproduction in any medium, provided you give appropriate credit to the original author(s) and the source, provide a link to the Creative Commons license, and indicate if changes were made. The Creative Commons Public Domain Dedication waiver (http://creativecommons.org/publicdomain/zero/1.0/) applies to the data made available in this article, unless otherwise stated. 
brief pre-patent period means that exposure to infective stages between cestocidal treatments can lead to infection and development of adult tapeworms in dogs. Dog owners easily underestimate the required frequency of cestocidal re-treatment administration needed to prevent $D$. caninum infections from reaching the egg shedding stage. Re-infection of pets can occur very quickly following cestocidal treatment, which has no residual effect [9].

Effective and persistent flea control can control the environmental proglottid load and prevent $D$. caninum infection, provided that fleas are killed sufficiently quickly before animals with fleas become tapeworm infected [4]. This is an additional benefit from an effective flea control regimen, adding to the control of other flea-related disorders such as flea-bite dermatitis and flea hypersensitivity dermatitis [2, 9]. PCR assessment found that $2.2 \%$ of $C$. felis from client-owned cats and $5.2 \%$ of C. felis from client-owned dogs in Europe were D. caninum infected [7]. These results show that the prevalence of $D$. caninum in Europe is sufficient to ensure that appropriate measures are routinely needed to prevent this tapeworm infection of domestic dogs and cats.

Fluralaner (Bravecto Chews and Bravecto Spot-On, Merck Animal Health, Madison, NJ, USA) is a highly effective flea insecticide that is systemically distributed in dogs following either topical or oral administration [13-15]. This active ingredient kills fleas following ingestion of a blood meal, with an onset of activity within two hours of initial oral administration [13]. Flea insecticidal efficacy following oral fluralaner administration reaches 98$100 \%$ at $8-24$ hours after flea infestation [13] and efficacy of $\geq 99 \%$ has been demonstrated for 12 weeks following application of a single topical dose of fluralaner [13, 16, 17]. The hypothesis in this study is that fluralaner treatment will provide flea insecticidal efficacy that is sufficiently rapid to prevent D. caninum transmission to dogs infested with infected fleas. This result was previously reported in dogs treated orally with a combination of another isoxazoline class molecule, afoxolaner, and milbemycin against challenges with infected fleas over a 28-day study period [18]. The current study evaluated both orally and topically administered fluralaner at the recommended clinical dose $(25-56 \mathrm{mg} / \mathrm{kg})$ with flea challenges over the 12 week period following a single treatment. Fluralaner does not have a label indication against cestodes.

\section{Methods}

This study was a parallel group designed, blinded, randomized, single center, placebo controlled efficacy study [19]. The study consisted of 24 dogs within 3 groups of 8 dogs each from an initial enrolled group of 28 dogs. Dogs were either beagles or mongrels (mixed-breed), and body weight ranged between $12.0-27.6 \mathrm{~kg}$, with a mean body weight of $17.7 \mathrm{~kg}$ prior to commencement of the study (Day -3). Mean body weight within the groups was $17.7 \mathrm{~kg}$ in Group 1, $17.1 \mathrm{~kg}$ in Group 2 and $17.6 \mathrm{~kg}$ in Group 3. No statistically significant differences were recorded with respect to the body weights $(P=0.9640)$ measured in different groups, indicating homogeneity at the time of inclusion. There were four male and four female dogs within each group ranging from 12 to 85 months-old at the time of inclusion. To be included in the study, dogs had to have been clinically healthy on physical examination by a veterinarian on Day -7 , older than 6 months of age at the time of inclusion, not clinically pregnant and not of an excessively fractious temperament which makes handling overtly difficult. The four dogs with the lowest body measurements on Day -2 were excluded from the study. Dogs included in the study had not been treated with a long-acting acaricide/insecticide during the 12 weeks preceding Day 0, and were also not treated with a macrocyclic lactone or other long acting anthelmintic during the three weeks preceding Day 0 (with the exception of deworming with a short-acting anthelmintic (a combination of praziquantel, pyrantel pamoate and febantel) during the study preparation phase prior to Day -7). None of the dogs were removed from the study prior to scheduled study termination and after inclusion on Day -3 (with the exception of the dogs that were infected with $D$. caninum).

Study dogs were acclimatized to conditions for 21 days before treatment and a centrifuged fecal parasite examination was run on all dogs on the first day of acclimatization to ensure the dogs were free of resident enteric parasites. The centrifuged fecal examination was conducted by thoroughly mixing the entire fecal sample of each dog to ensure a homogenized sample after collection. One gram of the homogenized sample was mixed with $10 \mathrm{ml}$ of sugar solution and strained through a double layer of gauze. A $15 \mathrm{ml}$ centrifuge tube was filled with the suspension and placed into the centrifuge and the tube was filled with sugar solution to a slight positive meniscus. A coverslip was placed on top of each tube, simultaneously ensuring that a small bubble was present under the coverslip. Samples were centrifuged in a swinging-head centrifuge at $1250 \times \mathrm{rpm}$ for $5 \mathrm{~min}$. After centrifugation, the tubes were removed and placed in a test tube rack and let stand for 10 min, then coverslips were removed and examined. All dogs were weighed and treated with a cestocide, a combination of milbemycin oxime and praziquantel (Milbemax ${ }^{\circ}$, Elanco, Greenfield, IN, USA). Their cages were screened daily for proglottids over the following 20-day acclimation period to detect any resident tapeworm infections persisting despite treatment.

Two days before treatment administration, dogs were ranked within sex in descending order of individual body weights, and blocked into 3 groups of 8 dogs each. One group was treated topically with sterile water, another 
group received orally administered fluralaner and the third received topically administered fluralaner. Fluralaner was dosed according to the product label, at a dose of $25-56 \mathrm{mg} / \mathrm{kg}$ body weight. Dogs treated with oral fluralaner were also treated with topical sterile water to maintain blinding. On treatment day, all dogs received half of their daily food ration approximately $20 \mathrm{~min}$ before treatment and the second half directly after treatment. All dogs were observed hourly for $6 \mathrm{~h}$ after treatment administration.

Thirty fleas per batch were sampled from at least three batches of fleas and examined microscopically for the presence of $D$. caninum metacestodes (Table 1) to determine the proportion containing the infective stage [20]. Approximately $100 \mathrm{D}$. caninum-infected C. felis fleas were placed on every dog in the study at $7,14,21,28$, $35,42,49,56,63,70,77$ and 83 days after treatment.

Visual proglottid inspections and counts were conducted daily on cage floors, sleeping areas and dog feces of all dogs from 35 to 113 days post-treatment to detect cestode-infected dogs. All dogs observed to have shed proglottids, and all dogs at the end of the study period, were removed from the study, dewormed and treated with a flea adulticide.

The experimental unit was the individual dog and the $D$. caninum infection incidence at the end of the study period was calculated for each group using the formula:

Infection incidence $(\%)=($ No. of dogs infected in each group / No. of dogs enrolled in each group) $\times 100$.

Significance was determined by comparing the infection incidence in each of the treated groups with the sterile water-treated control group (SAS Version 9.3 TS Level 1M2). Proportions were compared between the groups using a Fisher's exact test. Significance of the two sided significance test was set at $5 \%$.

\section{Results}

All 8 dogs in the placebo treated control group were observed to be shedding $D$. caninum proglottids: there were 3 positive control dogs at 35 days after treatment, 1 positive control dog at 38 days after treatment, and 4 positive control dogs at 43 days after treatment. Transmission of $D$. caninum to all control dogs confirms that the challenge is adequate. No dog in either of the fluralaner-treated groups shed $D$. caninum proglottids at any time during the post-treatment observation period between 35 and 113 days post-treatment. Therefore, both oral and topically administered fluralaner were $100 \%$ effective for prevention of transmission of D. caninum tapeworms to dogs in this natural flea infestation model. This difference between the proportion of $D$. caninum-infected dogs in the control and treated groups was significant (Fisher's exact test, $P<0.0001$ ) (Table 2).

\section{Discussion}

These results show that treatment with either topically or orally administered fluralaner kills fleas with sufficient rapidity to prevent transmission of $D$. caninum to dogs throughout the 84-day period following administration of a single dose. The overall study period extended to 113 days to allow maturation of any $D$. caninum possibly infecting the intestinal tracts of dogs. This result is consistent with the flea control observed following oral fluralaner administration to dogs in field challenge situations [16, 21, 22] and in laboratory challenges $[13,22]$. The fluralaner onset of activity following oral

Table 1 Dipylidium caninum infection in flea batches used to infest dogs using a natural challenge model

\begin{tabular}{|c|c|c|c|c|}
\hline Time post-treatment (days) & Flea age (days) & $\begin{array}{l}\text { Metacestode infection } \\
\text { prevalence }(\%)^{\mathrm{a}}\end{array}$ & $\begin{array}{l}\text { Metacestode intensity } \\
\text { per infected flea }^{\mathrm{b}}\end{array}$ & Metacestode abundance \\
\hline 7 & 12 & 23.3 & 3.1 & 0.7 \\
\hline 14 & 14 & 66.7 & 8.8 & 5.8 \\
\hline 21 & 13 & 33.3 & 3.3 & 1.1 \\
\hline 28 & 18 & 40.0 & 2.8 & 1.1 \\
\hline 35 & 14 & 13.3 & 6.8 & 0.9 \\
\hline 42 & 14 & 13.3 & 5.0 & 0.7 \\
\hline 49 & 14 & 16.7 & 2.0 & 0.3 \\
\hline 56 & 12 & 13.3 & 1.0 & 0.1 \\
\hline 63 & 14 & 13.3 & 1.3 & 0.2 \\
\hline 70 & 14 & 26.7 & 5.1 & 1.4 \\
\hline 77 & 16 & 13.3 & 9.3 & 1.2 \\
\hline 84 & 13 & 13.3 & 3.5 & 0.5 \\
\hline
\end{tabular}

${ }^{\mathrm{a}}$ No. infected fleas / total no. fleas examined

bTotal no. metacestodes recovered / no. infected fleas

'Total no. metacestodes recovered / total no. of fleas examined 
Table 2 Dipylidium caninum infection incidence for dogs treated and subsequently challenged with D. caninum infected Ctenocephalides felis

\begin{tabular}{lllll}
\hline Treatment & $\begin{array}{l}\text { Sterile } \\
\text { water }\end{array}$ & $\begin{array}{l}\text { Topical } \\
\text { fluralaner }\end{array}$ & $\begin{array}{l}\text { Oral } \\
\text { fluralaner }\end{array}$ & $P_{\text {-value }}$ \\
\hline $\begin{array}{l}\text { No. of proglottid-shedding } \\
\text { dogs per } 8 \text { dog group }\end{array}$ & 8 & 0 & 0 & $<0.0001$ \\
$\begin{array}{l}\text { Dipylidium caninum infection } \\
\text { prevention efficacy }\end{array}$ & na & $100 \%$ & $100 \%$ & \\
\hline
\end{tabular}

${ }^{a}$ For each treated group compared to the placebo-treated control using Fisher's exact test

Abbreviation: na - not applicable

administration to dogs is rapid, with mortality observed at 1 hour after dosing; significant flea mortality compared with untreated control dogs at 2 hours; and $99.4 \%$ mortality of adult fleas by 8 hours of dosing [13].

The $D$. caninum challenge presented to dogs in this study was greater than might be encountered under natural conditions. Field survey data in Europe indicate that $5.2 \%$ of fleas infesting client-owned dogs are infected with D. caninum [7], while in this study, all dogs were experimentally infested with approximately 100 fleas at weekly intervals, with 13 to $68 \%$ of the challenge fleas estimated to be D. caninum-infected (Table 1). Dipylidium caninum can also be transmitted by biting lice (Trichodectes canis), a transmission mechanism not addressed in the present study [23] although field efficacy has been shown for fluralaner against the sucking louse, Linognathus setosus [24].

Flea challenges began 7 days after treatment and continued until 83 days after treatment. Housing and dog feces were examined daily for $D$. caninum proglottids from 35 days until 113 days (30 days after the final flea challenge) after treatment. The intervals between the initial flea challenge on day 7 , the start of the observation period on day 35 , the final flea challenge (day 83 ) and the end of the study (day 113) provided time to allow the parasite to complete its pre-patent period in any infected dog and begin shedding proglottids. No proglottids were seen in the feces of any fluralaner treated dog, regardless of the route of administration, at any time point. Detection of $D$. caninum proglottids in feces, cage floor and bedding is sufficiently sensitive to detect infected dogs [18].

The cysticercoid larvae of D. caninum require 24-36 hours following arrival of the flea on the dog to become infective for the definitive host. These results confirm that either oral or topical administration of fluralaner effectively kills fleas before this $D$. caninum development time has elapsed and is consistent with the reported speed of kill times for orally administered fluralaner over the recommended retreatment interval [13].

\section{Conclusions}

The insecticidal efficacy of a single treatment of either orally or topically administered fluralaner prevented $D$. caninum transmission from infected fleas to susceptible dogs for up to 12 weeks following administration.

\section{Abbreviation}

PCR: polymerase chain reaction

\section{Acknowledgements \\ The authors are sincerely grateful to the Research Operations team and to all the monitors who ensured adherence to GCP standards.}

Funding

This study was funded by MSD Animal Health (Madison NJ, USA).

Availability of data and materials

All data generated or analyzed during this study are included in this published article.

\section{Authors' contributions}

All authors assisted in the study design, monitoring, interpretation of results, manuscript preparation and data analysis. All authors read and approved the final manuscript.

\section{Ethics approval and consent to participate}

The present research was approved and complied with the Clinvet International Institutional Animal Care and Use Committee. Ethics approval was granted by the Institutional Animal Care and use Committee (IACUC), members of which had the authority to inspect the study site and animals at will.

Consent for publication

Not applicable.

\section{Competing interests}

DG is currently employed by MSD Animal Health, Sydney, Australia and RA is currently employed by Merck Animal Health, Madison, NJ, USA. LM and JS worked on this project under contract with Merck Animal Health.

\section{Publisher's Note}

Springer Nature remains neutral with regard to jurisdictional claims in published maps and institutional affiliations.

\section{Author details}

${ }^{1}$ MSD Animal Health, 26 Talavera Rd, Macquarie Park, NSW 2113, Australia. ${ }^{2}$ Clinvet International, Uitzich Road, Bainsvlei, Bloemfontein 9338, South Africa. ${ }^{3}$ Merck Animal Health, 2 Giralda Farms, Madison, NJ 07940, USA.

Received: 7 August 2018 Accepted: 10 October 2018

Published online: 25 October 2018

\section{References}

1. Guzman RF. A survey of cats and dogs for fleas: with particular reference to their role as intermediate hosts of Dipylidium caninum. N Z Vet J. 1984;32:71-3.

2. Dryden MW, Rust MK. The cat flea: biology, ecology and control. Vet Parasit. 1994:52:1-19.

3. Rust M. The biology and ecology of cat fleas and advancements in their pest management: a review. Insects. 2017:8:118.

4. Beugnet F, Delport P, Luus H, Crafford D, Fourie J. Preventive efficacy of Frontline ${ }^{\oplus}$ Combo and Certifect ${ }^{\oplus}$ against Dipylidium caninum infestation of cats and dogs using a natural flea (Ctenocephalides felis) infestation model. Parasite. 2013;20:7

5. Pugh RE, Moorhouse DE. Factors affecting the development of Dipylidium caninum in Ctenocephalides felis felis (Bouché, 1835). Parasitol Res. 1985;71:765-75.

6. Pugh RE. Effects on the development of Dipylidium caninum and on the host reaction to this parasite in the adult flea (Ctenocephalides felis felis). Parasitol Res. 1987;73:171-7.

7. Beugnet F, Labuschagne M, Fourie J, Jacques G, Farkas R, Cozma V, et al. Occurrence of Dipylidium caninum in fleas from client-owned cats and dogs in Europe using a new PCR detection assay. Vet Parasit. 2014;205:300-6.

8. Mani I, Maguire JH. Small animal zoonoses and immuncompromised pet owners. Top Companion Anim Med. 2009;24:164-74. 
9. Fourie JJ, Crafford D, Horak IG, Stanneck D. Prophylactic treatment of fleainfested dogs with an imidacloprid / flumethrin collar (Seresto ${ }^{\oplus}$, Bayer) to preempt infection with Dipylidium caninum. Parasitol Res. 2013;112:33-46.

10. Chappell C, Enos JP, Pen HM. Dipylidium caninum, an underrecognised infection in infants and children. Pediatr Infect Dis J. 1990;9:745-7.

11. Raitiere CR. Tapeworm (Dipylidium caninum) infestation in a 6-month-old infant. J Fam Prac. 1992;34:101-2.

12. de Avelar DM, Bussolotti AS, Ramos M, Linardi PM. Endosymbionts of Ctenocephalides felis felis (Siphonaptera: Pulicidae) obtained from dogs captured in Belo Horizonte, Minas Gerais, Brazil. J Invertebr Pathol. 2007:94:149-52.

13. Taenzler J, Wengenmayer C, Williams H, Fourie J, Zschiesche E, Roepke RK, et al. Onset of activity of fluralaner (BRAVECTO ${ }^{\mathrm{TM}}$ ) against Ctenocephalides felis on dogs. Parasit Vectors. 2014;7:567.

14. Kilp S, Ramirez D, Allan MJ, Roepke RK, Nuernberger MC. Pharmacokinetics of fluralaner in dogs following a single oral or intravenous administration. Parasit Vectors. 2014;7:85

15. Kilp S, Ramirez D, Allan MJ, Roepke RK. Comparative pharmacokinetics of fluralaner in dogs and cats following single topical or intravenous administration. Parasit Vectors. 2016;9:296.

16. Meadows C, Guerino F, Sun F. A randomized, blinded, controlled USA field study to assess the use of fluralaner topical solution in controlling canine flea infestations. Parasit Vectors. 2017;10:36.

17. Taenzler J, Gale B, Zschiesche E, Roepke RKA, Heckeroth AR. The effect of water and shampooing on the efficacy of fluralaner spot-on solution against Ixodes ricinus and Ctenocephalides felis infestations in dogs. Parasit Vectors. 2016;9:233.

18. Beugnet F, Meyer L, Fourie J, Larsen D. Preventive efficacy of NexGard Spectra ${ }^{\oplus}$ against Dipylidium caninum infection in dogs using a natural flea (Ctenocephalides felis) infestation model. Parasite. 2017;24:16.

19. Marchiondo AA, Holdsworth PA, Fourie LJ, Rugga D, Hellmannd K, Snyder DE, et al. World Association for the Advancement of Veterinary Parasitology (W.A.A.V.P.) second edition: Guidelines for evaluating the efficacy of parasiticides for the treatment, prevention and control of flea and tick infestations on dogs and cats. Vet Parasit. 2013;194:84-97.

20. Venard CE. Morphology, bionomics, and taxonomy of the cestode Dipylidium caninum. Ann N Y Acad Sci. 1937;3:273-328.

21. Rohdich N, Roepke RK, Zschiesche E. A randomized, blinded, controlled and multi-centered field study comparing the efficacy and safety of Bravecto ${ }^{\text {TM }}$ (fluralaner) against Frontline ${ }^{\text {TM }}$ (fipronil) in flea- and tick-infested dogs. Parasit Vectors. 2014;7:83.

22. Williams $H$, Young DR, Qureshi T, Zoller $H$, Heckeroth AR. Fluralaner, a novel isoxazoline, prevents flea (Ctenocephalides felis) reproduction in vitro and in a simulated home environment. Parasit Vectors. 2014;7:275.

23. Saini VK, Gupta S, Kasondra A, Rakesh RL, Latchumikanthan A. Diagnosis and therapeutic management of Dipylidium caninum in dogs: a case report. J Parasit Dis. 2016;40:1426-8.

24. Kohler-Aanesen H, Saari S, Armstrong R, Péré K, Taenzler J, Zschiesche E, et al. Efficacy of fluralaner (Bravecto ${ }^{\text {TM }}$ chewable tablets) for the treatment of naturally acquired Linognathus setosus infestations on dogs. Parasit Vectors. 2017;10:426

\section{Ready to submit your research? Choose BMC and benefit from:}

- fast, convenient online submission

- thorough peer review by experienced researchers in your field

- rapid publication on acceptance

- support for research data, including large and complex data types

- gold Open Access which fosters wider collaboration and increased citations

- maximum visibility for your research: over $100 \mathrm{M}$ website views per year

At $\mathrm{BMC}$, research is always in progress.

Learn more biomedcentral.com/submissions 